\title{
COMPLICAÇÕES URETERAIS NOS PACIENTES ADULTOS SUBMETIDOS A TRANSPLANTE RENAL INTERVIVOS NO SERVIÇO DE UROLOGIA DA SANTA CASA DE SÃO PAULO (101 CASOS)
}

\author{
Ureteral complications after living donor kidney transplantation at Santa Casa of São Paulo \\ Luiz Renato Montez Guidoni', Fernando Korkes', Roni de Carvalho Fernandes², Álvaro Bosco ${ }^{3}$, Rafael Scherer ${ }^{3}$, \\ Marcio Rosa Pagan 4 , Christian Fuhro ${ }^{5}$, Marjo Cardenuto Perez ${ }^{6}$
}

\begin{abstract}
RESUMO:
Introdução: Dentre as complicações ureterais, as mais comuns são fístula, estenose e refluxo, incidindo em cerca de 2 a $10 \%$ dos pacientes transplantados. Objetivo: Avaliar a incidência das complicações ureterais dos transplantes renais intervivos de adultos. Métodos: Foi realizado estudo retrospectivo de 101 casos de transplante renal intervivos realizados em adultos, na Santa Casa de São Paulo, entre Jun/1977 e Mar/2005. Destes, 60 eram homens e 41, mulheres, com média de idade de 36 anos. Resultados: Houve nove casos de complicações ureterais $(8,9 \%)$, sendo um caso de estenose, sete casos de fístula urinária e um caso de obstrução ureteral por cálculo. Não houve casos de refluxo. Em seis casos, as complicações foram precoces e ocorreram dentro dos trinta dias iniciais do pós-operatório. Destes casos, sete ocorreram nos 14 anos iniciais de transplante, e após 1991 houve apenas dois casos de complicações ureterais. Conclusão: As complicações ureterais do transplante renal intervivos em adultos foram eventos pouco freqüentes $(8,9 \%)$ em nossa casuística, e ocorreram principalmente nos primeiros anos de transplante.
\end{abstract}

Descritores: complicações; ureter; transplante; renal; intervivos; adulto.

Disciplina de Urologia do Departamento de Cirurgia da Santa Casa de São Paulo.

1. Residente de Urologia da Santa Casa de São Paulo, Membro aspirante da Sociedade Brasileira de Urologia.

2. Mestre em Urologia pela Santa Casa de São Paulo, Professor assistente da disciplina de urologia da Santa Casa de São Paulo, Membro Titular da Sociedade Brasileira de Urologia, Membro associado da Associação Brasileira de Transplante de Órgãos.

3. Residente de Cirurgia Geral da Santa Casa de São Paulo.

4. Assistente da disciplina de Urologia da Santa Casa de São Paulo, Membro associado da Associação Brasileira de Transplante de Órgãos

5. Mestre em Urologia pela Santa Casa de São Paulo, Assistente da disciplina de Urologia da Santa Casa de São Paulo, Membro Titular da Sociedade Brasileira de Urologia, Membro associado da Associação Brasileira de Transplante de Órgãos.

6. Professor Doutor em Urologia pela Santa Casa de São Paulo, chefe da disciplina de Urologia e Professor Adjunto do Departamento de Cirurgia da Santa Casa de São Paulo, Membro da Sociedade Brasileira de Urologia.

Endereço para Correspondência: Luiz Renato Montez Guidoni

Av. Lacerda Franco 206, Cambuci - São Paulo - SP - Cep 01536000

Email: guidoni@medscape.com - Telefax: 1132095553.

Recebido em:13/06/05 Aceito em:05/07/05

\section{INTRODUÇÃO}

Dentre todos os transplantes de órgãos sólidos, o renal é o mais realizado, tendo havido mais de 10000 nos EUA no último ano'. Há 40 anos foi realizado o primeiro transplante renal com sucesso, sendo este o tratamento de escolha para os pacientes com insuficiência renal terminal. Apesar desse tempo, ainda apresentamse muitas complicações dessas cirurgias, quando comparadas com outros procedimentos do mesmo porte cirúrgico. Dentre essas complicações, as urológicas são as mais freqüentes, variando entre 2,5 a $15 \%$ acarretando morbidade significativa, podendo afetar a evolução da sobrevida do enxerto. ${ }^{2,3,4}$

As complicações ureterais nos transplantes renais tiveram sua incidência reduzida nos últimos 20 anos segundo a literatura mundial. ${ }^{5}$ Atribui-se tal fato principalmente às evoluções em técnicas operatórias e às terapias de imunossupressão desenvolvidas nos últimos tempos. ${ }^{6}$ Dentre as complicações ureterais, as mais comuns são: fístula, estenose e refluxo. ${ }^{7}$

\section{OBJETIVO}

Avaliar a incidência das complicações ureterais dos transplantes renais intervivos de adultos.

\section{MÉTODOS}

Foi realizado estudo retrospectivo de 101 casos consecutivos de transplante renal intervivos realizado em adultos, no serviço de transplante da Santa Casa de Misericórdia de São Paulo, 
entre junho de 1977 e março de 2005. Do total de pacientes transplantados, 60 eram do sexo masculino e 41 do feminino, com média etária de 36 anos. A anastomose vésico-ureteral (técnica extra-vesical) foi realizada após a revascularização do enxerto. Os casos que apresentaram complicações ureterais foram analisados individualmente e em relação aos possíveis fatores relacionados.

\section{RESULTADOS}

Dos 101 pacientes submetidos a transplante renal intervivos houve nove casos de complicações ureterais $(8,9 \%)$. Dos pacientes que apresentaram complicações, sete eram do sexo masculino (77,7\%) e dois do feminino (23,3\%), com média de idade de 37 anos. Dos nove casos de complicações ureterais, houve um caso de estenose $(0,9 \%)$, sete casos de fístula urinária $(6,9 \%)$ e um caso de obstrução ureteral por cálculo $(0,9 \%)$ (Figura 1$)$.

Figura 1.

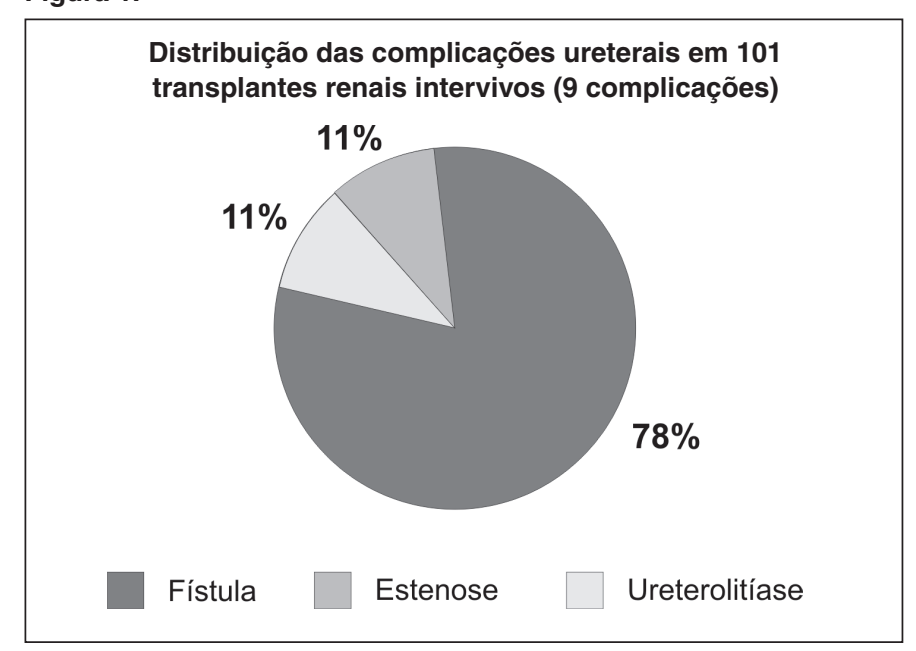

Não houve casos de refluxo vésico-ureteral. Em seis casos, as complicações foram precoces e ocorreram dentro dos trinta dias iniciais do pós-operatório. Destes casos, sete ocorreram nos 14 anos iniciais de transplante, e após 1991 houve apenas dois casos de complicações ureterais, o que pode ser explicado pela melhora das técnicas de imunossupressão, pelas melhores técnicas cirúrgicas e pela melhora na curva de aprendizado. (Figura 2)

Figura 2.

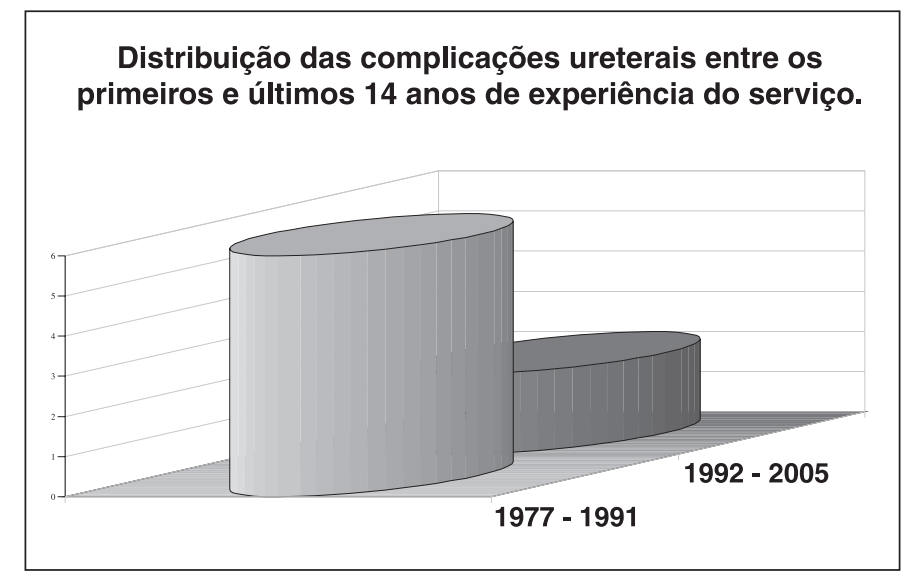

\section{DISCUSSÃO}

A incidência de fístulas urinárias oscila entre $1 \%$ e $15 \%$ e os percentuais de mortalidade relacionada às fístulas oscila entre $13 \%$ e $100 \%{ }^{8,9,10,11}$ Essas fístulas têm múltiplas causas: insuficiência vascular, tipo de técnica cirúrgica empregada para restabelecer o trato urinário, surto de rejeições, infecções urinárias e no local do enxerto e hematoma no local do enxerto, entre outras. ${ }^{12}$

A obstrução ureteral tem incidência que varia entre $0,3 \%$ a 7,5\% e pode ser precoce (secundária a edema e hematoma locais, linfocele ou isquemia ureteral), ou tardia (devido à fibrose retroperitoneal, compressão extrínseca e isquemia crônica do ureter por episódios repetidos de rejeição). ${ }^{13,14,15}$

A respeito de cálculos ureterais, os estudos demonstram que a incidência não chega a $1,5 \%$ dos casos de transplante renal. ${ }^{16}$

Entre os trabalhos mais expressivos, temos um estudo com 1000 transplantes, com incidência de $3,6 \%$ de obstrução ureteral e $2,5 \%$ de fístula, além de $0,2 \%$ de litíase ureteral e $0,1 \%$ de refluxo vesicoureteral. Em todos os casos, a complicação não levou a dano permanente do enxerto. ${ }^{13}$ Já em outro estudo mais recente com 1787 transplantes, é relatada obstrução ureteral de 4,1\%, sem alteração na sobrevida do paciente e do enxerto e sem descrição do número de fístulas. ${ }^{17}$

Alguns trabalhos relatam melhora no índice de complicações após o emprego de cateter duplo J na anastomose ureterovesical. O mais importante deles é uma metanálise com sete trabalhos controlados e randomizados, com 1154 pacientes, onde se concluiu que o uso do cateter reduz significantemente as complicações ureterais maiores; em contra partida, houve perda de dois enxertos $(0,17 \%)$ por infecção do trato urinário, além de maior incidência desta quando não utilizado cotrimoxazole $480 \mathrm{mg} / \mathrm{d}$. Os autores ainda salientam que cateteres maiores $(>20 \mathrm{~cm})$ deixados por um período de tempo superior a seis semanas, têm maior possibilidade de migração e criação de crostas ao redor deste. ${ }^{18}$ Ao encontro destes dados, um estudo iraniano relata $14 \%$ de fístulas urinárias com anastomose ureterovesical com técnica intravesical e $4,4 \%$ com técnica extravesical e apenas $0,4 \%$ após o uso do cateter. ${ }^{19}$ Outro exemplo é o estudo indiano que relata $8,5 \%$ de complicações ureterais sem o uso do cateter, com diminuição para $0,22 \%$ após o uso deste. ${ }^{20}$ Outros dois estudos defendem essa tese: o primeiro com 248 pacientes demonstrou $12 \%$ de complicações ( $7 \%$ de fístula e $5 \%$ de obstrução) sem o emprego de cateter duplo J e $2 \%$ (todas fístulas) após o uso do cateter e outro com 194 transplantes realizados, diminuiu de $6 \%$ para $1 \%$ o número de fístulas ureterais e de $4 \%$ para $0 \%$ as obstruções após o emprego do cateter. Esse último demonstrou que a incidência de infecção urinária e a sobrevida do enxerto são as mesmas em ambos os grupos. ${ }^{21,22}$ Em nosso serviço, por motivo de custo (o cateter não é coberto pelo sistema de saúde) e pela baixa morbidade das complicações ureterais relatadas acima, o cateter não é utilizado rotineiramente.

\section{CONCLUSÃO}

As complicações ureterais do transplante renal intervivos em adultos foram eventos pouco freqüentes em nossa casuística, compatíveis com dados da literatura e ocorreram principalmente nos primeiros anos de transplante. 


\section{ABSTRACT}

Introduction: Among urethral complications, the most common are fistula, stenosis and reflux, happening in about 2 to $10 \%$ of transplanted patients. Objective: The aim of the present study was to analyze the urethral complications in the living donor kidney transplantation. Method: A retrospective study of 101 kidney-transplanted patients (60 male, 41 female) at Santa Casa of São Paulo between 1977 and 2005 was performed. Results: From an amount of 9 urethral complications $(8.9 \%)$, there was 1 case of urethral stenosis, 7 cases of urinary fistula, and 1 case of urethral obstruction due to urethral calculi. There were no cases of vesicourethral reflux. In 6 cases, early complications were diagnosed within the first month after surgery. From all cases, 7 happened in the 14 initial years of kidney transplantations, and after 1991, there were only 2 cases reported. Conclusion: Urethral complications of living donor renal transplantation in an adult population was a rare event in our experience $(8.9 \%)$, and most of them often observed during the first years of the experience in our institution.

Key words: complications; ureter; kidney transplantation; renal; living donor; adult.

\section{REFERÊNCIAS}

1. Cecka JM, Terasaki PI. Clinical Transplants 1995. 1995;:1-18.

2. Lapointe, SP; Charbit, M; Jan, D; Lortat-Jacob, S; Michel, JL; Beurton, D; et al.: Urological complications after renal transplantation using ureteroureteral anastomosis in children. J Urol, 166: 1046-1048, 2001.

3. Cimic, J; Meuleman, EJ; OOsterhof, GO; et al.: Urological complication in the renal transplantation. A comparison between living-related and cadaveric grafts. Eur Urol, 31:433, 1997.

4. Kashi, SH, Lodge, JP, Giles, GR; et al.: Ureteric complications of renal transplantation. Br J Urol, 70: 139, 1992.

5. Makisalo H, Eklund B, Salmela K, Isoniemi H, Kyllonew L, Hockerstedt K. Urological complications after 2084 consecutive kidney transplants. Transplant Proc 1997;299:152-153

6. Nahas,W.C. - Transplante renal com doador "cadáver": relação de aspectos do doador, do receptor e do ato cirúrgico com a evolução do doente. São Paulo, 1993. ( Tese - Doutorado - Faculdade de Medicina da Universidade de São Paulo ).

7. Taylor, RJ; Murillo, D. Renal transplantation: surgical aspects, complications and results. Curr Opin Urol 8:135-140 1998 Rapid Science Ltd.

8. Martin. D.C.; Mims, M.M.; Kaufman, J.J.; Goodwin, W.E. - The ureter in renal transplantation. J. Urol., 101:680-7, 1969

9. Hobart, MG; Streem, SB; Gill, IS: Renal transplant complications. Urol Clin Nor Am 27:787-798, 2000.

10. Matalon, TA; Thompson, MJ; Patel,SK; et al.: Percutaneous treatment of urine leaks in renal transplantation patients. Radiology 174:1049-1051, 1990

11. Barroso Junior, U; Lipay, MAS; D’ávila, CLR.; Picanço Neto, JM; Abbud Filho, M Zerati Filho, M: Complicações cirúrgicas pós-transplantes renais. Análise de 356 casos. J. Bras. Urol., 23:71-6 1997

12. Brandina, L. - Estudo comparativo de fístula urinária no transplante renal com anastomose pielo-piélica e com ureteroneocistostomia extra-vesical. São Paulo, 1982.
(Tese - Livre Docência - Faculade de Medicina da Universidade de São Paulo).

13. Shoskes. D.A.; Hanbury, D.; Cranston,D.; Morris, P,J. - Urological complications in 1000 consective renal transplant recipients. J. Urol., 153:18-21, 1995.

14. Leary, F.J.; Woods, H.E.; Deweerd, J.H - Urologic problems in renal transplantatio. Arch. Surg., 110:1124-6, 1975.

15. Lamaster, D.; Katzberg, R.W.; Confer, D.J.; Slaysman, M.L. - Ureteropelvic fibrosis in renal transplants: radiographic manifestation. AJR Am. J. Roentgenol., 135:79-82, 1980.

16. Hayes ,JM; Streem, SB; Graneto, D; et al.: Renal transplant calculi: A re-evaluation of risks and management. Transplantation 47:949-952,1989.

17. Karam, G; Hetet, J.-F; Maillet, F; Rigaud, J; Hourmant, M; Soulillou, J.-P; Giral, M: Late Ureteral Stenosis Following Renal Transplantation: Risk Factors and Impact on Patient and Graft Survival. Am J Transp 6(2):352-356, 2006

18. Wilson CH, Bhatti AA, Rix DA, Manas DM: Routine intraoperative ureteric stenting for kidney transplant recipients. Cochrane Database Syst Rev. 2005 Oct 19;(4): CD004925.

19. Taghavi, R; Mahdavi, R; Khmmar, MA; Darabi, MR: Living renal transplantation and urological complications in 13 years' experience in one center. Br J Urol 90(2): 215-216, 2002.

20. Kumar, A; Verma, BS; Srivastava, A; Bhandari, M; Gupta, A; Sharma, R: Evaluation of the urological complications of living related renal transplantation at a single center during the last 10 years: impact of the double-j stent. J. Urol., 164(3):657-660, 2000 .

21. Butterworth, PC; Horsburgh, T; Veitch, P S; Bell, PRF; Nicholson, ML: Urological complications in renal transplantation: impact of a change of technique. Br J Urol 79(4): 499-502, 1997.

22. Benoit, G, Blanchet, $P$, Eschwege, $P$ et al: Insertion of a double pigtail ureteral stent for the prevention of urological complications in renal transplantation: a prospective randomized study. J Urol, 156:881, 1996. 